\title{
BMJ Open Efficacy of clarithromycin in patients with mild COVID-19 pneumonia not receiving oxygen administration: protocol for an exploratory, multicentre, open-label, randomised controlled trial (CAME COVID-19 study)
}

\author{
Kazuko Yamamoto (D) , ${ }^{1,2}$ Naoki Hosogaya, ${ }^{3}$ Noriho Sakamoto (i) , \\ Haruo Yoshida, ${ }^{4}$ Hiroshi Ishii, ${ }^{5}$ Kazuhiro Yatera (D) , ${ }^{6}$ Koichi Izumikawa, ${ }^{7}$ \\ Katsunori Yanagihara, ${ }^{8}$ Hiroshi Mukae ${ }^{1}$
}

To cite: Yamamoto $\mathrm{K}$, Hosogaya N, Sakamoto N, et al. Efficacy of clarithromycin in patients with mild COVID-19 pneumonia not receiving oxygen administration: protocol for an exploratory, multicentre, open-label, randomised controlled trial (CAME COVID-19 study). BMJ Open 2021;11:e053325. doi:10.1136/ bmjopen-2021-053325

- Prepublication history and additional supplemental material for this paper are available online. To view these files, please visit the journal online (http://dx.doi.org/10.1136/ bmjopen-2021-053325)

Received 11 May 2021 Accepted 31 August 2021

Check for updates

(C) Author(s) (or their employer(s)) 2021. Re-use permitted under CC BY-NC. No commercial re-use. See rights and permissions. Published by BMJ.

For numbered affiliations see end of article.

Correspondence to Dr Kazuko Yamamoto; kazukomd@nagasaki-u.ac.jp

\section{ABSTRACT}

Introduction The COVID-19 pandemic has emerged worldwide. Although several medications have been approved for treating moderate-to-severe COVID-19, very few treatment strategy has been established for patients with mild COVID-19 who do not require oxygen administration. Clarithromycin is a macrolide antimicrobial agent that has been widely used for bacterial respiratory infectious diseases. Clarithromycin also acts an immunomodulating drug and suppresses cytokine storms in viral respiratory diseases, including influenza. In this study, we aim to evaluate the efficacy of clarithromycin in patients with mild COVID-19.

Methods and analysis This is an exploratory, multicentre, open-label, randomised controlled trial. This study was initiated in May 2021 and will end in July 2022. Patients with mild COVID-19 pneumonia who do not require oxygen administration will be enrolled and randomly assigned in a 1:1:1 ratio to group $A$ (administration of clarithromycin $800 \mathrm{mg} /$ day), group B (administration of clarithromycin $400 \mathrm{mg} /$ day) or group C (standard treatment without clarithromycin). The planned number of enrolled patients is 60 ( 20 patients $\times$ three groups). The primary endpoint is the number of days required to improve the clinical symptoms as measured by the severity score. Secondary endpoints include days for recovery of the body temperature, proportion of patients with oxygen administration, inflammatory cytokines, viral load, serum immunoglobulins, peripheral blood lymphocytes, blood biomarkers and pneumonia infiltrations.

Ethics and dissemination The study protocol was approved by the Clinical Research Review Board of Nagasaki University in accordance with the Clinical Trials Act in Japan. The study will be conducted in accordance with the Declaration of Helsinki, the Clinical Trials Act and other current legal regulations in Japan. Written informed consent will be obtained from all the participants. The results of this study will be reported as journal publications. Trial registration number $\mathrm{jRCTs} 071210011$.
Strengths and limitations of this study

- This is the first randomised controlled trial to evaluate the efficacy of clarithromycin in COVID-19 pneumonia, especially in patients with mild COVID-19 pneumonia who do not require oxygen administration.

- The results of this study could contribute to the development of new treatment strategies for COVID-19 pneumonia.

- The major limitations of this study are its exploratory nature and relatively small sample size.

- Another limitation is the open-label study design and generalisability since this study is conducted only in Japan in Japanese patients.

\section{INTRODUCTION}

The COVID-19 pandemic is currently a major concern worldwide. In Japan, a cumulative of 932361 PCR test-positive cases have been confirmed, and 15190 deaths were reported by the Ministry of Health, Labour and Welfare in Japan as of 1 August 2021. ${ }^{1}$ Approximately $5 \%$ of the patients with COVID-19 were hospitalised, $1.6 \%$ had severe symptoms requiring intensive care and $1.0 \%$ died in Japan. ${ }^{2}$ Recently, dexamethasone and remdesivir have been used as standard treatments for patients with moderate-to-severe COVID-19 who require respiratory support, ${ }^{3-5}$ and the monoclonal antibody therapy, such as casirivimab/imdevimab antibody cocktail, have been demonstrated as effective for mild to moderate COVID-19. ${ }^{6-8}$ However, these treatments require intravenous drip infusion, and no oral medical treatment has been established for mild COVID-19, which accounts 
for the majority (approximately 80\%) of patients with COVID-19.

The mechanism of exacerbation in COVID-19 has been reported to correlate with dysregulation of the immune response, resulting in exaggerated inflammation to produce excessive cytokines (the so-called cytokine storm). ${ }^{9}$ Indeed, infection with the SARS-CoV-2 induces high expression of inflammatory cytokines, such as granulocyte macrophage colony-stimulating factor and interleukin-6 (IL-6), thereby accelerating the inflammation. ${ }^{10}$ Therefore, suppression of inflammatory cytokines is an important target for preventing the exacerbation of COVID-19. This is supported by the evidence that tocilizumab, an antihuman IL-6 receptor monoclonal antibody that inhibits IL-6 signalling, and dexamethasone, anti-inflammatory and immunosuppressing steroid, reduced risk of mortality and exacerbation that required ventilation. ${ }^{11-13}$

Clarithromycin is a macrolide antibiotic that has been widely used as a monotherapy for bacterial respiratory infectious diseases. Clarithromycin has also been used as a standard combination therapy with beta-lactam antibiotics for severe community-acquired pneumonia, ${ }^{14}$ owing to its ability to suppress inflammatory cytokines. ${ }^{15} 16$ Viral respiratory diseases, such as influenza, are not an exception in the mechanism of exacerbation, and combination therapy with clarithromycin and antiviral agents demonstrated clinical efficacy in influenza A infection. ${ }^{17} 18$ Considering the use of macrolides for COVID19 , evidence in the efficacy of azithromycin to COVID-19 is controversial; some reported the beneficial effect of azithromycin on COVID-19, ${ }^{19}{ }^{20}$ while others reported no benefit in patients with COVID-19. ${ }^{21-23}$ Clarithromycin may have several advantages over azithromycin. First, clarithromycin is well tolerated, with even lower frequency of adverse events (AEs)/side effects compared with azithromycin. ${ }^{2425}$ Second, dose of clarithromycin can be adjusted based on patients' age, weight and symptoms by using a tablet of $200 \mathrm{mg}$ in Japan. Third, clarithromycin and azithromycin affect differently to suppress immune cells and inflammatory cytokine production, ${ }^{26}{ }^{27}$ and to inhibit NF- $\kappa \mathrm{B}$ activation. ${ }^{28}$ Together with these, clarithromycin is a good candidate for alleviating symptoms and preventing the exacerbation of COVID-19 by suppressing inflammatory cytokines and could be safely used in patients with COVID-19. This trial is planned to estimate the efficacy of clarithromycin in patients with mild COVID-19 pneumonia who do not require oxygen administration.

\section{METHODS AND ANALYSIS \\ Study design and setting}

'The CAM (Clarithromycin) Effectivity for COVID-19 pneumonia that does not require oxygen administration; multicenter, randomized-controlled, open-label, 3-armed parallel group comparison, exploratory trial' (CAME COVID study) was initiated in May 2021, following the approval by the Clinical Research Review Board in Nagasaki University in March 2021 and the registration/publication at the Japan Registry of Clinical Trials (jRCT) (registration number: jRCTs071210011) in April 2021. This study is scheduled to culminate in July 2022. Patients have been enrolled since May 2021, and enrolment will culminate in February 2022. This study will be conducted in 10 medical institutions in Japan (Nagasaki University Hospital, Nagasaki Harbor Medical Center, Sasebo City General Hospital, Japan Community Health Care Organization JCHO Isahaya General Hospital, Japanese Red Cross Nagasaki Genbaku Hospital, Hospital of the University of Occupational and Environmental Health, Japan, Wakamatsu Hospital of the University of Occupational and Environmental Health, Japan, Kitakyushu City Yahata Hospital, Fukuoka University Chikushi Hospital, and Saiseikai Nagasaki Hospital). As shown in figure 1, patients who are eligible for this study will be asked to participate in this study, and informed consent will be obtained prior to the registration/randomisation. After written consent is obtained from the eligible patients, they will be enrolled and randomised into group A (administration of clarithromycin $800 \mathrm{mg} /$ day), group B (administration

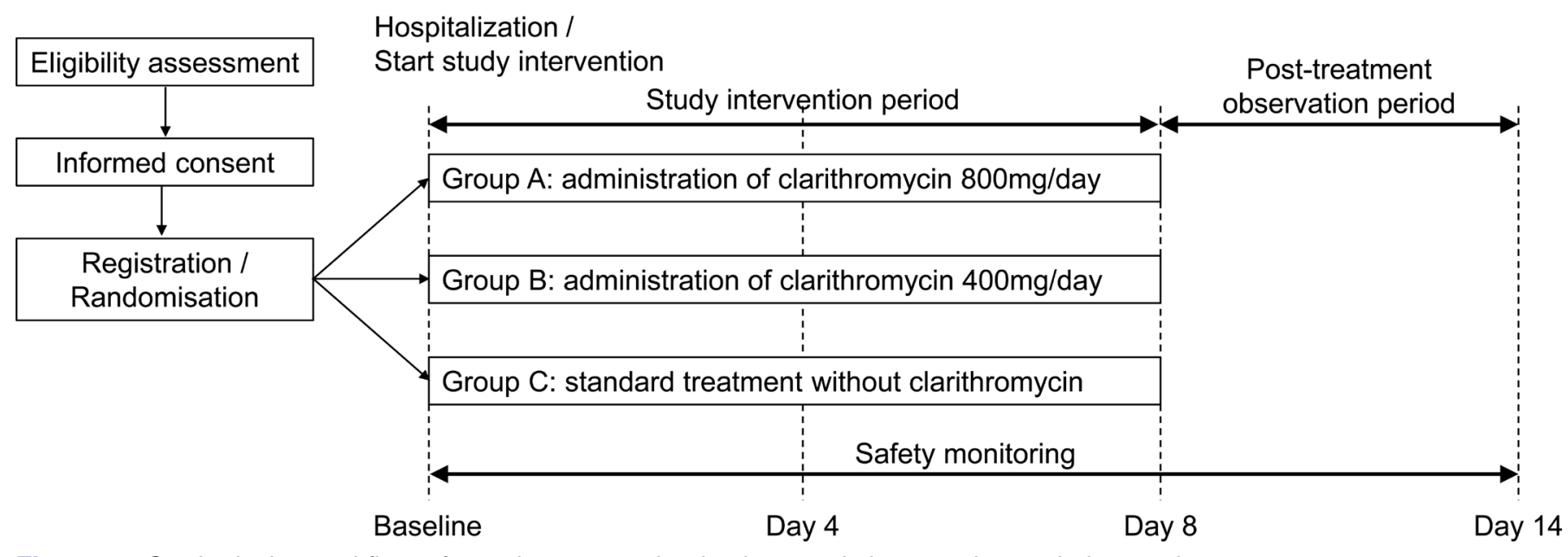

Figure 1 Study design and flow of recruitment, randomisation, study intervention and observation. 
of clarithromycin $400 \mathrm{mg} /$ day) or group C (standard treatment without clarithromycin). The rationale for the doses of clarithromycin is as follows: clarithromycinnaproxen-oseltamivir combination therapy for Influenza A using $1000 \mathrm{mg} /$ day of clarithromycin $(500 \mathrm{mg}$ twice daily) was tolerated and reduced mortality and length of hospital stay, ${ }^{17}$ and $400 \mathrm{mg} /$ day and $800 \mathrm{mg}$ /day of clarithromycin have been approved for bacterial respiratory infectious diseases and non-tuberculous mycobacterial infection, respectively, in Japan, and the safety of these doses of clarithromycin has been confirmed.

\section{Sample size calculation}

Since no prior clinical trial has evaluated the effect of clarithromycin on COVID-19 pneumonia, no reference data are available for the statistical sample size calculation for this study. Therefore, this study is planned as an exploratory trial, and the target number of enrolled patients is defined as 60 (20 subjects $\times 3$ groups), based on the possible number of patients who could give their consent during the planned enrolment period in this study at the participating medical institutions.

\section{Eligibility criteria}

Patients with mild COVID-19 pneumonia who do not require oxygen administration will be included in this study. Mild COVID-19 is defined as SARS-CoV-2 positive and percutaneous arterial oxygen saturation $\left(\mathrm{SpO}_{2}\right)$ of $94 \%$ or higher in this study. This study will enrol patients with mild COVID-19 and with pneumonia. The detailed inclusion criteria are as follows: (1) patients in whom SARS-CoV-2 is detected by PCR tests or loop-mediated isothermal amplification method within 3 days before the informed consent, (2) patients with pneumonia by routine chest radiography or chest CT, (3) Japanese patients who are aged 20 years or older and (4) patients who give their written consent for participating in the study. The exclusion criteria are as follows: (1) patients who had symptoms of 8 days or longer, (2) patients who were treated with macrolide antimicrobial agents, (3) patients who were treated with steroids (except inhalants) or immunosuppressive agents, (4) patients who are diagnosed with influenza viral infection, (5) patients whose $\mathrm{SpO}_{2}$ is $93 \%$ or less under room air condition, (6) patients with hepatic dysfunction (aspartate aminotransferase or alanine aminotransferase is more than five times the upper limit of normal in each medical institution or Child-Pugh B or C), (7) patients with renal dysfunction (creatinine is more than twice the upper limit of normal in each medical institution, and estimated glomerular filtration rate is less than $30 \mathrm{~mL} / \mathrm{min} / 1.73 \mathrm{~m}^{2}$ ), (8) patients whose peripheral blood neutrophils are less than $1000 / \mu \mathrm{L}$, (9) patients who have a history of hypersensitivity to macrolide antimicrobial agents, (10) patients who are pregnant or breast feeding, (11) patients who have a history of vaccination against COVID-19 and (12) patients with other conditions that the investigator thinks may render them inappropriate to participate in the study.

\section{Recruitment and consent}

The informed consent document (see online supplemental file 1) would be provided to the candidates who meet all of the inclusion criteria and who do not forsake any of the exclusion criteria to provide a comprehensive explanation of this study. Written consent will be obtained. After obtaining consent, the participants will be enrolled in this study.

\section{Random allocation}

After obtaining informed consent, eligible patients are randomly assigned in a 1:1:1 ratio to group A (administration of clarithromycin $800 \mathrm{mg} /$ day), group B (administration of clarithromycin $400 \mathrm{mg}$ /day) or group C (standard treatment without clarithromycin). The randomisation sequence is generated using a computer-based dynamic allocation method with a minimisation procedure to balance the allocation factors (age $\geq 20$ years and less than 40 years, $\geq 40$ years and less than 60 years or $\geq 60$ years) and sex.

\section{Study intervention and observation}

All enrolled patients in this study will be hospitalised, since the Infectious Diseases Control Law in Japan designated COVID-19 as category II infectious diseases, which warrants the isolation of the patients, and the COVID-19 infectious disease treatment guidelines by the Ministry of Health, Labour and Welfare in Japan ${ }^{29}$ warrant hospitalisation of the patients of COVID-19 with pneumonia. The enrolled patients will be subsequently observed for 7 days ${ }^{30}$ for a comprehensive follow-up during the intervention. Patients assigned to group A will commence with $400 \mathrm{mg}$ of clarithromycin orally twice daily (after breakfast and supper) following hospitalisation. Patients assigned to group B will commence with $200 \mathrm{mg}$ of clarithromycin orally twice daily (after breakfast and supper) following hospitalisation. Patients assigned to group $\mathrm{C}$ will commence with the standard care for COVID-19 pneumonia in each medical institution without the administration of clarithromycin following hospitalisation. Since this is an open-label trial, placebo is not used in the group C. In groups A and B, clarithromycin is administered 14 times (twice daily for 7 days). If the administration starts on the morning of day 1 (the day of hospitalisation), clarithromycin will be administered until the evening of day 7. If the administration starts on the evening of day 1 , clarithromycin will be administered until the morning of day 8 . The study patients are observed on days $1,2,3$, $4,5,6,7,8$ and 14 . The day of hospitalisation is considered the day when observation starts (reference date). During the study intervention period (days 1 to 7 or 8 ), the patients are not allowed to use macrolide antimicrobial agents, immunosuppressive agents or anticancer agents. Following the completion of the study intervention (day 8), the study patients will be discharged. After 
day 8 (post-treatment observation period), all the study patients will receive standard care for COVID-19 pneumonia in each medical institution without the administration of clarithromycin.

Table 1 shows the schedule of assessments performed at each observation point, including the mandatory and optional assessments. Inspection of subjects' characteristics (height, weight, body mass index, onset of COVID19, detection date of SARS-CoV-2, date of hospitalisation, anamnesis and comorbidity), vital signs (body temperature, systolic blood pressure, diastolic blood pressure, pulse, percutaneous oxygen saturation and frequency of breath), haematology tests (red blood cell, haemoglobin, haematocrit, white cell count, neutrophil, lymphocyte, eosinophil, monocyte, basophil and platelet), general blood biochemical tests (total bilirubin, aspartate aminotransferase, alanine aminotransferase, alkaline phosphatase, gamma-guanosine triphosphate, total cholesterol, total protein, albumin, blood urea nitrogen, creatinineestimated glomerular filtration rate based on creatinine, lactate dehydrogenase, creatine phosphokinase, brain natriuretic peptide, troponin $\mathrm{T}, \mathrm{C}$ reactive protein, procalcitonin, ferritin, $\mathrm{Na}, \mathrm{pH}$, haemoglobin A1c and glucose), blood coagulation tests (prothrombin time, activated partial thromboplastin time and d-dimer), chest radiography and CT, and inspection of medications of other pharmaceutical agents are conducted by general inspection and interview. The severity of COVID-19 pneumonia is measured by the severity classification according to the COVID-19 infectious disease treatment guidelines by the Ministry of Health, Labour and Welfare in Japan ${ }^{29}$ (mild: $\mathrm{SpO}_{2} \geq 96 \%$, no respiratory symptoms or only with coughing without dyspnoea and without pneumonia findings; moderate I: $93 \%<\mathrm{SpO}_{2}<96 \%$, with dyspnoea and with pneumonia findings; moderate II: $\mathrm{SpO}_{2} \leq 93 \%$ and oxygen administration required; and severe: admission to intensive care unit or ventilator required), pneumonia severity inde $\mathrm{x}^{31}$ and A-DROP defined in the guidelines for the management of community-acquired pneumonia in adults released from the Japanese Respiratory Society, ${ }^{32}$ the quantity of oxygen administered are recorded daily during hospitalisation, PCR tests for SARS-CoV-2 are conducted using nasopharyngeal swabs, 11) nasal drip tests are conducted for interleukin (IL) 1beta, IL-6, IL-8, IL-10, IL-17, tumour necrosis factor-alpha, interferongamma, beta-defensin, granulocyte-macrophage colonystimulating factor and immunoglobulin A, special blood tests are conducted for cytokines, chemokines, IL-33, immunoglobulin $\mathrm{M}$, immunoglobulin $\mathrm{G}$, and immunoglobulin A, medication adherence of the study agent, meal intake, and subjective symptoms measured by the severity score ${ }^{33}$ are daily answered on the study patients' diary by the study patients themselves.

\section{Outcomes}

The primary endpoint of this study is the number of days required to improve clinical symptoms, measured by the severity score ${ }^{33}$ by $50 \%$ or more from baseline.
Secondary endpoints are as follows: (1) days to recover the body temperature to below $37^{\circ} \mathrm{C}$ following registration, (2) change in inflammatory cytokines in serum and nasal discharge from baseline, (3) proportion of patients in whom all the clinical symptoms measured by the severity score completely disappeared, (4) proportion of patients in whom each clinical symptom measured by the severity score is improved by $50 \%$ or more from baseline, (5) reduction rate of the SARS-CoV-2 from baseline, (6) change in serum immunoglobulin G, immunoglobulin $\mathrm{M}$ and immunoglobulin $\mathrm{A}$ antibodies from baseline, (7) recovery rate of peripheral blood lymphocytes, (8) proportion of patients with oxygen administration, (9) change in other general blood biomarkers and vital signs from baseline and (10) change in pneumonia image by chest radiography or chest CT. The severity score ${ }^{33}$ is measured by enquiring the effect of seven pneumoniarelated symptoms (cough, shortness of breath, fatigue, headaches, anosmia, dysgeusia and general unwellness) on the patients' daily life on a 4-point Likert scale $(0=$ not affected, 1=little affected, $2=$ affected and $3=$ severely affected), and the number of days required to decrease the total score of the five symptoms by $50 \%$ or more from baseline will be analysed for the primary endpoint. The questionnaire for the severity score is shown in the online supplemental table 1.

\section{Data collection, data management and monitoring}

A case report form is used for data collection. A central registration number is used to identify the participants for anonymisation. Data collection and management are carried out by third-party entities to avoid bias. Data management is performed by Soiken, Data Management Group (Data Center). To manage and ensure quality, the study is monitored by Soiken, Monitoring Group.

\section{Safety evaluation}

During this study, the investigators constantly monitor any AEs through regular medical checkups. All the related AEs, not only side effects to the study agents but also any abnormal clinical laboratory test values and any untoward medical occurrence, are reported and documented. If the AEs meet the following criteria, the events are referred to as serious adverse events (SAEs) based on the ICH E2A, ICH E2D and the 'Ethical Guidelines for Medical and Health Research Involving Human Subjects' in Japan ${ }^{34}$ : (1) AEs that result in death, (2) AEs that are life-threatening, (3) AEs that require hospitalisation or prolongation of existing hospitalisation, (4) AEs that result in persistent or significant disability or incapability, (5) other AEs that are medically important or critical, (6) AEs that are equivalently severe to criteria (1) to (5) and (7) AEs that are congenital abnormality or birth defects. AEs are followed up until normalisation or recovery to a level not considered to be an AE. The first hospitalisation following the registration to day 8 and prolongation of the first hospitalisation after day 8 without worsening of pneumonia-related symptoms are not considered 


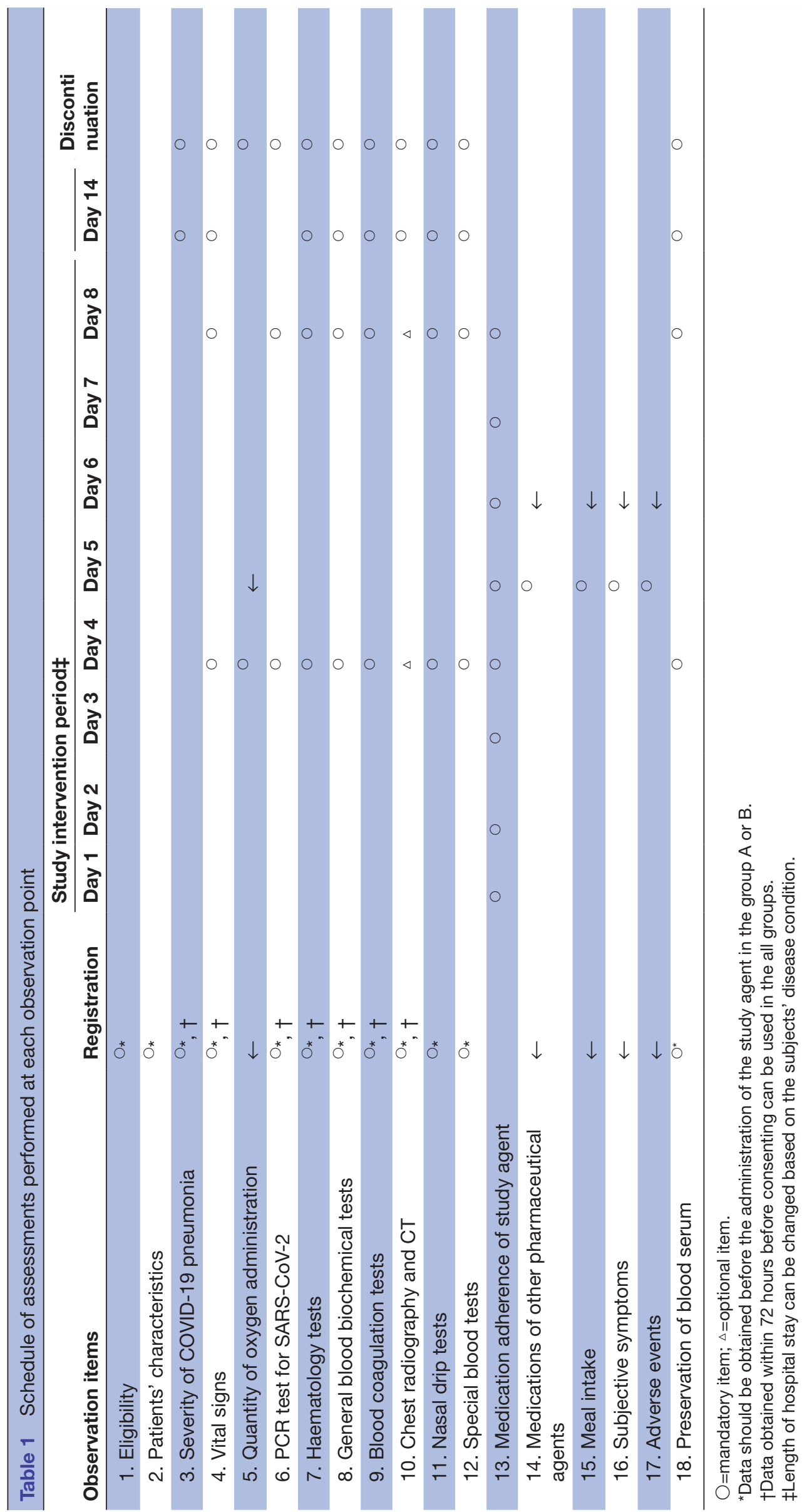

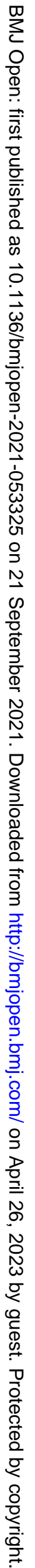


as AEs/SAEs in this study, since this study enrolled patients of COVID-19 with pneumonia who should be treated by hospitalisation until the complete disappearance of pneumonia-related symptoms, according to the COVID-19 infectious disease treatment guidelines by the Ministry of Health, Labour and Welfare in Japan. ${ }^{29}$

\section{Statistical analysis}

All the tests will be two sided, and a p value of $<0.05$ will be considered statistically significant. A statistical analysis plan will be developed prior to the database lock. All the statistical analyses will be conducted by independent biostatisticians.

Three analysis sets are defined in this study; the full analysis set (FAS) included all the patients who are registered in this study and assigned to one of the intervention groups. However, patients with a severe protocol violation, such as registration without consent or registration out of the enrolment period, will be excluded from the FAS. The per-protocol set (PPS) excludes patients with a protocol violation from the FAS, such as violation of eligibility criteria, use of prohibited or restricted concomitant drugs, or poor adherence to the study agent (less than $75 \%$ or more than $120 \%$ ). The safety analysis set includes all the patients who are registered in this study and receive at least one dose of the study agent or control treatment.

Patient characteristics at baseline will be presented as frequencies and proportions for categorical data, and summary statistics (number of patients, mean, SD, 95\% CI for mean, minimum, the first quartile, median, the third quartile and maximum) for continuous data. Patient characteristics will then be compared using the $\chi^{2}$ test or Fisher's exact test for categorical endpoints and analysis of variance or Kruskal-Wallis test for continuous variables.

The primary endpoint of this study is the number of days required to improve the clinical symptoms measured by the severity score ${ }^{33}$ by $50 \%$ or more from the baseline. The primary endpoint will be analysed using the FAS and PPS. The cumulative incidence curves for the days required to improve the clinical symptoms measured by the severity score will be drawn, and a log-rank test with a closed testing procedure will be conducted for comparison among the three groups. In addition, HRs among the groups will be estimated using the Cox proportional hazard model, and a $95 \% \mathrm{CI}$ will be calculated. The HR adjusted by allocation factors (age and sex) as covariates will also be estimated.

For the secondaryendpoints, summarystatistics (number of patients, mean, SD, $95 \%$ CI for mean, minimum, the first quartile, median, the third quartile and maximum) for measurements, changes from baseline and per cent changes from baseline will be calculated for continuous data. Frequencies and proportions will be calculated for the categorical data. Two-sample t-test or Wilcoxon rank sum test for intergroup comparisons of the continuous data, one-sample t-test or Wilcoxon signed-rank test for intragroup comparisons of the continuous data and the $\chi^{2}$ test or Fisher's exact test for intergroup comparisons of the categorical data will be performed. Multiplicity will not be adjusted for the secondary endpoints.

For the safety endpoints, summary statistics for the frequency of AEs will be calculated, and Fisher's exact tests will be performed for intergroup comparisons. Multiplicity will not be adjusted for the safety endpoints.

\section{Patient and public involvement}

The patients and the public are not involved in the study, including planning, execution, analysis and evaluation.

\section{Ethics and dissemination}

This study and its protocol were approved by the Clinical Research Review Board of Nagasaki University (approval no. CRB20-027) in accordance with the Clinical Trials Act of Japan. The study will be conducted in accordance with the Declaration of Helsinki, the Clinical Trials Act and other current legal regulations in Japan. Written informed consent will be obtained from all participants after a full explanation of the study. The results of this study will be disseminated at medical conferences and through journal publications.

\section{DISCUSSION}

This is the first randomised controlled trial to evaluate the efficacy of clarithromycin against COVID-19 pneumonia. Although several in vitro or bioinformatics analyses have reported the positive effect of macrolide antimicrobial agents on coronaviruses, ${ }^{35}$ evidence in the efficacy of azithromycin to COVID-19 is still controversial, ${ }^{19}{ }^{20}$ and no clear clinical evidence has confirmed the efficacy of clarithromycin on coronaviruses, especially SARS-CoV-2. Although recent studies demonstrated the usefulness of monoclonal antibody therapy for mild to moderate COVID- $19,{ }^{6-8}$ no treatment strategy has yet been established to prevent exacerbation in patients with mild COVID-19 who do not require oxygen administration. In addition, since the monoclonal antibodies need to be administered to the patients by drip infusion, oral drug treatment could have an advantage, especially for persons receiving treatment or waiting for treatment at home or non-medical accommodation facilities. Development of a proper treatment strategy for patients with mild COVID-19 is important, considering the medical aspects and in social and economic aspects, such as, for example, to reduce the hospital bed occupancy by patients with COVID-19 exacerbation or to suppress the treatment costs for patients with severe COVID-19. Clarithromycin may also contribute to the prevention of secondary infections owing to its antimicrobial/antiviral effects. Therefore, this study aims to evaluate the efficacy of clarithromycin in patients with mild COVID-19 pneumonia who do not require oxygen administration. The results of this study could contribute to the development of new treatment strategies for COVID-19 pneumonia. 
Our study has several limitations. First, it is exploratory in nature. Owing to the lack of previous clinical evidence that evaluated the effect of clarithromycin on COVID-19 pneumonia, the target number of enrolled patients is defined based on the feasible number of patients who can give their consent during the planned enrolment period in this study at the participating medical institutions. Second, the target sample size is relatively small. Third, this is an open-label trial. Thus, bias for the patients and investigators/physicians cannot be completely avoided. Fourth, this study is conducted only in medical institutions in Japan and enrols only Japanese patients. These constraints could limit the generalisability of this study. Further international clinical trials on a larger scale are required in the future.

\section{Author affiliations}

${ }^{1}$ Department of Respiratory Medicine, Nagasaki University Hospital, Nagasaki, Japan

${ }^{2}$ Clinical Research Center, National Organization Hospital Nagasaki Medical Center, Omura, Japan

${ }^{3}$ Clinical Research Center, Nagasaki University Hospital, Nagasaki, Japan

${ }^{4}$ Department of Otolaryngology-Head and Neck Surgery, Nagasaki University Graduate School of Biomedical Sciences, Nagasaki, Japan

${ }^{5}$ Department of Respiratory Medicine, Fukuoka University Hospital, Fukuoka, Japan ${ }^{6}$ Department of Respiratory Medicine, University of Occupational and Environmental Health, Kitakyushu, Japan

${ }^{7}$ Infection Control and Education Center, Nagasaki University Hospital, Nagasaki, Japan

${ }^{8}$ Department of Laboratory Medicine, Nagasaki University Hospital, Nagasaki, Japan

Acknowledgements The authors would like to thank all the clinical staff for their assistance in the execution of the study and Soiken for their technical assistance in the launch and execution of the study.

Contributors KYam and NH contributed to the conception and design of the study, drafted the protocol and supervised the revision. NS, HY, KO, HI, KYat, KI and KYan provided intellectual input to improve the study design and revise the protocol. HM supervised the conception and design of this study. All the authors read and approved the final manuscript.

Funding This study is financially supported by the Taisho Pharmaceutical. Award/ Grant number is not applicable. Taisho Pharmaceutical is not involved in this study, including planning, execution, data management, statistical analysis, evaluation or write-up.

Competing interests KYat has received grants from Bayer Yakuhin and has received honoraria for scientific lecture presentations, speaker bureaus, manuscript writing or educational events from AstraZeneca K.K., Kyorin Pharmaceutical, Boehringer Ingelheim Pharmaceuticals, GlaxoSmithKline K.K. and Novartis Pharma K.K. HM has received grants from Taisho Pharmaceutical, Boehringer Ingelheim Pharmaceuticals, Otsuka Pharmaceutical, Shionogi and Kyorin Pharmaceutical, has received honoraria for scientific lecture presentations, speaker bureaus, manuscript writing or educational events from Taisho Pharmaceutical, AstraZeneca K.K., Astellas Pharma, Shionogi, Daiichi Sankyo, Pfizer Japan, MSD K.K., Boehringer Ingelheim Pharmaceuticals and Kyorin Pharmaceutical and has received donation from FUJIFILM Toyama Chemical, Taiho Pharmaceutical, Chugai Pharmaceutical, Daiichi Sankyo, Meiji Seika Pharma, Kyorin Pharmaceutical and Shionogi. All funding agencies play no role in the study design, data collection and analysis, decision to publish, or preparation of the manuscript. The other authors declare no conflict of interest.

Patient consent for publication Not required.

Provenance and peer review Not commissioned; externally peer reviewed.

Supplemental material This content has been supplied by the author(s). It has not been vetted by BMJ Publishing Group Limited (BMJ) and may not have been peer-reviewed. Any opinions or recommendations discussed are solely those of the author(s) and are not endorsed by BMJ. BMJ disclaims all liability and responsibility arising from any reliance placed on the content. Where the content includes any translated material, BMJ does not warrant the accuracy and reliability of the translations (including but not limited to local regulations, clinical guidelines, terminology, drug names and drug dosages), and is not responsible for any error and/or omissions arising from translation and adaptation or otherwise.

Open access This is an open access article distributed in accordance with the Creative Commons Attribution Non Commercial (CC BY-NC 4.0) license, which permits others to distribute, remix, adapt, build upon this work non-commercially, and license their derivative works on different terms, provided the original work is properly cited, appropriate credit is given, any changes made indicated, and the use is non-commercial. See: http://creativecommons.org/licenses/by-nc/4.0/.

\section{ORCID iDs}

Kazuko Yamamoto http://orcid.org/0000-0002-1357-1676

Noriho Sakamoto http://orcid.org/0000-0002-7577-476X

Kazuhiro Yatera http://orcid.org/0000-0002-2955-4432

\section{REFERENCES}

1 Ministry of Health, Labour and Welfare in Japan. Novel coronavirus (COVID-19), 2021. Available: https://www.mhlw.go.jp/stf/ seisakunitsuite/bunya/0000164708_00079.html

2 Ministry of Health, Labour and Welfare in Japan. 11 knowledge of the "now" about the novel coronavirus infectious disease (August, 2021 version), 2021. Available: https://www.mhlw.go.jp/stf/seisakunitsuite/ bunya/0000121431_00256.html

3 Horby P, Lim WS, RECOVERY Collaborative Group. Dexamethasone in hospitalized patients with Covid-19 - preliminary report. N Engl J Med 2020;17:NEJMoa2021436.

4 Beigel JH, Tomashek KM, Dodd LE, et al. Remdesivir for the Treatment of Covid-19 - Final Report. N Engl J Med 2020;383:1813-26.

5 Jorgensen SCJ, Kebriaei R, Dresser LD. Remdesivir: review of pharmacology, pre-clinical data, and emerging clinical experience for COVID-19. Pharmacotherapy 2020;40:659-71.

6 Weinreich DM, Sivapalasingam S, Norton T, et al. REGN-COV2, a neutralizing antibody cocktail, in outpatients with Covid-19. N Engl J Med 2021;384:238-51.

7 Piccicacco N, Zeitler K, Montero J, et al. Effectiveness of severe acute respiratory syndrome coronavirus 2 monoclonal antibody infusions in high-risk outpatients. Open Forum Infect Dis 2021;8:ofab292.

8 Koehler J, Ritzer B, Weidlich S, et al. Use of monoclonal antibody therapy for nosocomial SARS-CoV-2 infection in patients at high risk for severe COVID-19: experience from a tertiary-care hospital in Germany. Infection 2021;in press. doi:10.1007/s15010-021-01657-y. [Epub ahead of print: 09 Jul 2021].

9 Channappanavar R, Perlman S. Pathogenic human coronavirus infections: causes and consequences of cytokine storm and immunopathology. Semin Immunopathol 2017;39:529-39.

10 Zhou Y, Fu B, Zheng X, et al. Pathogenic T-cells and inflammatory monocytes incite inflammatory storms in severe COVID-19 patients. Natl Sci Rev 2020;7:998-1002.

11 Hermine O, Mariette X, Tharaux P-L, et al. Effect of tocilizumab vs usual care in adults hospitalized with COVID-19 and moderate or severe pneumonia: a randomized clinical trial. JAMA Intern Med 2021:181:32-40.

12 Salama C, Han J, Yau L, et al. Tocilizumab in patients hospitalized with Covid-19 pneumonia. N Engl J Med 2021;384:20-30.

13 RECOVERY Collaborative Group, Horby P, Lim WS, et al. Dexamethasone in hospitalized patients with Covid-19. N Engl J Med 2021;384:693-704.

14 Kyriazopoulou E, Sinapidis D, Halvatzis S, et al. Survival benefit associated with clarithromycin in severe community-acquired pneumonia: a matched comparator study. Int $J$ Antimicrob Agents 2020;55:105836.

15 Demartini G, Esposti D, Marthyn P, et al. Effect of multiple doses of clarithromycin and amoxicillin on IL-6, IFNgamma and IL-10 plasma levels in patients with community acquired pneumonia. $J$ Chemother 2004;16:82-5.

16 Spyridaki A, Raftogiannis M, Antonopoulou A, et al. Effect of clarithromycin in inflammatory markers of patients with ventilatorassociated pneumonia and sepsis caused by gram-negative bacteria: results from a randomized clinical study. Antimicrob Agents Chemother 2012;56:3819-25.

17 Hung IFN, To KKW, Chan JFW, et al. Efficacy of clarithromycinnaproxen-oseltamivir combination in the treatment of patients hospitalized for influenza $A(\mathrm{H} 3 \mathrm{~N} 2)$ infection: an open-label randomized, controlled, phase Ilb/III trial. Chest 2017;151:1069-80. 
18 Yatera K, Umeki K, Yamasaki K, et al. The additive effect of clarithromycin on influenza A infection in the elderly patients and patients with comorbid diseases. Respir Investig 2017;55:380-3.

19 PRINCIPLE Trial Collaborative Group. Azithromycin for community treatment of suspected COVID-19 in people at increased risk of an adverse clinical course in the UK (principle): a randomised, controlled, open-label, adaptive platform trial. Lancet 2021;397:1063-74

20 Sekhavati E, Jafari F, SeyedAlinaghi S, et al. Safety and effectiveness of azithromycin in patients with COVID-19: an open-label randomised trial. Int J Antimicrob Agents 2020;56:106143.

21 Molina JM, Delaugerre C, Le Goff J, et al. No evidence of rapid antiviral clearance or clinical benefit with the combination of hydroxychloroquine and azithromycin in patients with severe COVID-19 infection. Med Mal Infect 2020;50:384.

22 Furtado RHM, Berwanger O, Fonseca HA, et al. Azithromycin in addition to standard of care versus standard of care alone in the treatment of patients admitted to the hospital with severe COVID-19 in Brazil (coalition II): a randomised clinical trial. Lancet 2020;396:959-67.

23 Cavalcanti AB, Zampieri FG, Rosa RG, et al. Hydroxychloroquine with or without azithromycin in mild-to-moderate Covid-19. N Engl J Med 2020;383:2041-52.

24 Müller O. Comparison of azithromycin versus clarithromycin in the treatment of patients with upper respiratory tract infections. $J$ Antimicrob Chemother 1993;31 Suppl E:137-46.

25 Bradbury F. Comparison of azithromycin versus clarithromycin in the treatment of patients with lower respiratory tract infection. $J$ Antimicrob Chemother 1993;31 Suppl E:153-62.

26 Sugiyama K, Shirai R, Mukae H, et al. Differing effects of clarithromycin and azithromycin on cytokine production by murine dendritic cells. Clin Exp Immunol 2007;147:540-6.
27 Mukae H, Urabe K, Yanagihara K, et al. Low expression of T-cell co-stimulatory molecules in bone marrow-derived dendritic cells in a mouse model of chronic respiratory infection with Pseudomonas aeruginosa. Tohoku J Exp Med 2010;220:59-65.

28 Morinaga Y, Yanagihara K, Miyashita N, et al. Azithromycin, clarithromycin and telithromycin inhibit MUC5AC induction by Chlamydophila pneumoniae in airway epithelial cells. Pulm Pharmacol Ther 2009;22:580-6.

29 Ministry of Health, Labour and Welfare in Japan. COVID-19 infectious disease treatment guidelines (ver.5.1), 2021. Available: https://www.mhlw.go.jp/stf/seisakunitsuite/bunya/0000121431_ 00111.html

30 Wang D, Hu B, Hu C, et al. Clinical characteristics of 138 hospitalized patients with 2019 novel coronavirus-infected pneumonia in Wuhan, China. JAMA 2020;323:1061-9.

31 Fine MJ, Auble TE, Yealy DM, et al. A prediction rule to identify lowrisk patients with community-acquired pneumonia. $N$ Engl J Med 1997;336:243-50.

32 Japanese Respiratory Society. The JRS guidelines for the management of community-acquired pneumonia in adults. Japanese Respiratory Society 2007 https://www.jrs.or.jp/modules/basicauth/ index.php?file=adult_pneumonia1-3.pdf

33 Janowitz T, Gablenz E, Pattinson D, et al. Famotidine use and quantitative symptom tracking for COVID-19 in non-hospitalised patients: a case series. Gut 2020;69:1592-7.

34 Ministry of Health, Labour and Welfare in Japan. Ethical guidelines for medical and health research involving human subjects, 2021. Available: https://www.mhlw.go.jp/stf/seisakunitsuite/bunya/ hokabunya/kenkyujigyou/i-kenkyu/index.html

35 Poddighe D, Aljofan M. Clinical evidences on the antiviral properties of macrolide antibiotics in the COVID-19 era and beyond. Antivir Chem Chemother 2020;28:204020662096171. 